\title{
EFEITOS DO PROTOCOLO DE ESTRESSORES SOBRE A PREFERÊNCIA DE ÁGUA COM SACAROSE EM RATOS
}

EFFECTS OF CHRONIC MILD STRESS ON THE PREFERENCE FOR SUCROSE SOLUTION IN RATS

PAULO EDUARDO DA SILVA

FANI ETA KORN MALERBI

PontifícIa Universidade CATÓlica de SÃo PaUlo, Brasil

\begin{abstract}
RESUMO
O presente estudo teve o objetivo de investigar os efeitos do Protocolo de Estressores (PE) sobre a preferência de água com sacarose em ratos submetidos a um regime contínuo de privação de água para manter seus pesos a $85 \%$ ad lib. Dez ratos machos foram submetidos a 13 testes de consumo de água e de água com sacarose. Desses, oito foram também submetidos a sessões operantes (Concorrente VIVI) e ao PE por seis semanas consecutivas, um sujeito foi submetido aos testes de consumo e ao PE e um sujeito apenas aos testes de consumo. Os resultados revelaram que a submissão ao PE não foi acompanhada por um decréscimo na preferência por água com sacarose $(\mathrm{p}>0,05)$, nem nos testes de consumo, nem nas sessões operantes, independentemente da condição experimental. Entretanto, observou-se uma diminuição significativa $(\mathrm{p}<0,05)$ no consumo de ração na gaiola viveiro enquanto os animais estavam sendo submetidos ao PE. Sugere-se que a redução no consumo de ração na gaiola viveiro seja uma medida alternativa para avaliar a diminuição do valor reforçador de um estímulo decorrente da exposição ao PE.

Palavras-chave: Estressores crônicos moderados, privação, modelo animal, esquema concorrente VIVI, anedonia.
\end{abstract}

\begin{abstract}
The goal of the present study was to investigate the effect of Chronic Mild Stress (CMS) on the preference for sucrose solution in rats at a continuous regimen of water deprivation in order to keep their weights at $85 \%$ ad lib. Ten male Wistar rats were submitted to 13 tests of consumption of water with and without sucrose, one of these subjects being exclusively exposed to those tests. Of the remaining subjects, one was also submitted to CMS for six consecutive weeks and besides that eight rats were also submitted to operant sessions (Concurrent VIVI). The results showed that the submission to CMS was not followed by a decrease $(p>0.05)$ in the preference for water with sucrose neither in the consumption tests, nor in operant sessions independently of the experimental condition. However, it was detected a significant decrease $(p<0.05)$ in food consumption at the cage where the rats lived when they were submitted to CMS. This reduction in the consumption of food at the cage when the animals were submitted to CMS suggests that this variable can be used as an alternative measure to evaluate the effects of CMS on the value of a reinforcing stimulus.

Key words: Chronic Mild Stress, deprivation, animal model, Concurrent VIVI schedule, anhedonia.
\end{abstract}

\footnotetext{
O presente estudo é parte da dissertação de mestrado do primeiro autor sob orientação da segunda autora, desenvolvido no Programa de Pós-Graduação em Psicologia Experimental: Análise do Comportamento da PUC-SP. Os autores agradecem à CAPES pelo financiamento parcial da pesquisa. Correspondência referente a esta artigo devem ser enviadas a Paulo Silva: pauloedu.s@gmail.com
}

DOI. 10.18542/rebac.v14i2.7530 
Modelos animais têm sido utilizados para reproduzir em laboratório condições análogas às observadas em doenças humanas (Antoniuk, Bijata, Ponimaskin, \& Wlodarczyk, 2019). Os termos em inglês Chronic Mild Stress (CMS) referem-se a um modelo animal de anedonia ${ }^{1}$ desenvolvido por Willner, Towell, Sampson, Sophokleous e Muscat (1987) com base no estudo de Katz (1982) que verificou que ratos expostos a um conjunto de estímulos estressores moderados e incontroláveis, que compunham um Protocolo de Estressores (PE), apresentaram um decréscimo no consumo de água adocicada em relação ao consumo apresentado antes da submissão a esse protocolo.

$\mathrm{O}$ primeiro dos quatro experimentos de Willner et al. (1987) - todos realizados com ratos da linhagem Lister - teve como objetivo investigar os efeitos do PE na preferência por uma solução de água com $1 \%$ de sacarina em relação à água pura em 24 ratos. Além de trabalhar com uma solução adocicada com sacarina, o segundo experimento avaliou também a preferência de água adocicada com sacarose numa concentração de $1 \%$ e acrescentou o objetivo de avaliar os efeitos do antidepressivo tricíclico denominado desmetilemipramina (DMI) em dois grupos de 10 ratos cada. O terceiro experimento, realizado com dois grupos de 11 ratos cada, teve os mesmos objetivos que o estudo 2, além de verificar os níveis de corticosterona (um glicocorticóide envolvido na resposta de estresse) e de glicemia (taxa de açúcar no sangue) nos sujeitos. O quarto experimento - com dois grupos de seis ratos cada também teve como objetivo verificar os efeitos do DMI sobre a preferência de água adocicada com sacarose, porém numa concentração menor $(0,6 \%)$. O conjunto de estressores variou nos diversos experimentos realizados por esses autores. Nos experimentos 1 e 4, os estressores utilizados foram: (a) privação de água e de ração; (b) iluminação contínua; (c) gaiola suja; (d) outro animal na gaiola; (e) inclinação da gaiola em $30^{\circ}$; (f) temperatura reduzida $\left(10^{\circ} \mathrm{C}\right)$. Nos experimentos 2 e 3 , os autores acrescentaram: (g) luz estroboscópica; (h) cheiro novo; (i) barulho intermitente; (j) exposição a uma garrafa vazia após um período de privação de água; (k) presença de um objeto estranho na caixa e (1) acesso restrito à ração na gaiola viveiro. No experimento 2, esses mesmos estressores foram utilizados, porém a intensidade destes foi aumentada gradualmente ao longo de seis semanas. $\mathrm{O}$ período de exposição ao $\mathrm{PE}$ foi de cinco, seis e nove semanas nos experimentos 1,2 e 3 , respectivamente. $\mathrm{O}$ quarto experimento não teve sua duração especificada pelos autores.

Os resultados mostraram que os sujeitos de Willner et al. (1987) submetidos ao PE apresentaram uma redução na preferência por água com sacarose de aproximadamente uma média de $9,5 \mathrm{~g}$ na semana zero para aproximadamente $4,0 \mathrm{~g}$ na semana cinco (conforme

\footnotetext{
${ }^{1}$ Anedonia é um sintoma central para o diagnóstico de depressão em humanos, descrita no Manual Diagnóstico e Estatístico de Transtornos Mentais (DSM-V) como um déficit na capacidade de sentir prazer e interesse pelas coisas
}

Figura 2 do artigo original) e uma redução de aproximadamente uma média de $15,0 \mathrm{~g}$ para 8,0 $\mathrm{g}$ de água com sacarina (conforme Figura 1 do artigo original), enquanto o consumo de água pura manteve-se em torno das médias 9,5 e $10 \mathrm{~g}$ ao longo do estudo (conforme Figura 2 do artigo original). No segundo e no terceiro experimentos, após duas semanas da administração do DMI, houve uma recuperação na preferência por água adocicada. Tanto os níveis de glicemia quanto os de corticosterona permaneceram inalterados ao longo do estudo. Nos testes de consumo, os sujeitos do quarto experimento apresentaram menor preferência pela solução com menor concentração de sacarose $(0,6 \%)$ do que pela solução com maior concentração (1\%). Willner et al. (1987) concluíram que a diminuição na preferência por água adocicada após a submissão ao PE não era produto de uma regulação calórica, pois essa redução ocorreu tanto com sacarose (substância calórica) quanto com sacarina (substância não calórica), nem decorrente da alteração dos níveis de corticosterona e de glicemia e hipotetizaram que o PE produziu uma insensibilidade à água adocicada o que poderia ser considerada um indicador de anedonia.

Inicialmente estudado por meio da resposta consumatória de beber num teste de preferência, os efeitos do PE também foram avaliados por meio de outras respostas consumatórias, tais como o consumo de água e de ração na gaiola viveiro (Almeida, 2013; Cardoso \& Banaco, 2009; Dolabela, 2004; Lacerda, 2013; Oliveira, 2009; Pereira \& Sério, 2010; Pereira, 2015; Rodrigues, 2005; Thomaz, 2009) e de classes de respostas instrumentais, como a taxa de respostas em esquemas de razão progressiva (Barr \& Phillips, 1998; Willner et al., 1998), em esquemas concorrentes de razão fixa (Dolabela, 2004; Pereira \& Sério, 2010; Rodrigues, 2005; Thomaz, 2001, 2009), em esquemas concorrentes de intervalo variável (Cardoso \& Banaco, 2009), em esquemas concorrentes de tempo variável (Almeida, 2013) e em esquemas múltiplos que alternaram reforçamento e extinção envolvendo esquema de reforçamento contínuo (Rocha, 2013) ou de razão fixa (Fonseca Júnior, Castelli, \& Oliveira, 2015) nos componentes de reforçamento. O estudo de respostas instrumentais foi sugerido por Willner et al. (1998) que criticaram as medidas consumatórias como um índice "confiável" de responsividade à recompensa.

De modo geral, os resultados apresentados na literatura podem ser agrupados em três categorias: 1) uma redução na preferência por água com sacarose nos testes de consumo e na taxa de respostas de pressão à barra reforçadas com água com sacarose após a submissão ao PE (Dolabela, 2004; Rodrigues, 2005; Thomaz, 2001), 2) uma redução na preferência por água com sacarose nos testes de consumo, sem alteração na taxa de respostas de pressão à barra reforçadas com água com sacarose (Barr \& Phillips, 1998; Cardoso \& Banaco, 2009; Oliveira, 2009) e 3) uma ausência de redução em ambas as medidas (Almeida, 2015; Fonseca Júnior et al., 2015; Pereira \& Sério, 2010; Rocha, 2013). 
O consumo de água e de ração na gaiola viveiro após a submissão ao PE variou nos diferentes estudos. Almeida (2013), Cardoso e Banaco (2009) e Rocha (2013) observaram um aumento no consumo médio $( \pm 10$ gramas) de ração na gaiola viveiro. Nos estudos de Rodrigues (2005), Oliveira (2009) e Thomaz (2009) o padrão de consumo permaneceu estável em comparação com a linha de base, ao passo que nos estudos de Lacerda (2013) e Pereira (2015) o consumo de ração diminuiu entre cinco e dez gramas para todos os sujeitos após a submissão ao PE. Pereira e Sério (2010) observaram diferentes padrões no consumo de água e de ração em função das manipulações nas variáveis privação de ração e de água em diferentes condições experimentais.

Nas pesquisas citadas, a privação de água e de ração eram também componentes do $\mathrm{PE}$, o que pode ter prejudicado a análise do efeito do PE sobre o consumo de água e de ração na gaiola viveiro, uma vez que a privação real à qual os animais foram submetidos era maior do que aquela descrita como um dos componentes do protocolo (i.e., $85 \%$ ad lib mais 24 horas de privação de água e de ração antes dos testes de consumo e períodos de até de 43 horas de privação de ração durante o protocolo).

Os dados da literatura sugerem que a variabilidade no consumo de água e de ração na gaiola viveiro observada nos estudos de Almeida (2013), Cardoso e Banaco (2009), Lacerda (2013), Oliveira (2009), Pereira e Sério (2010), Rodrigues (2005) e Thomaz (2009) talvez possa estar relacionada aos diferentes esquemas de privação adotados. Uma forma de evitar a sobreposição da privação anterior ao protocolo e o conjunto de privações (água e ração) previstas no PE, seria manter os animais em um regime único de privação durante todo o estudo, eliminando as privações de água e de ração do conjunto de estímulos estressores do protocolo. Isto poderia ajudar a elucidar os efeitos do PE nas medidas de consumo de água e de água com sacarose e no consumo diário de água e de ração na gaiola viveiro.

Uma análise das pesquisas que avaliaram o efeito do PE sobre a preferência por água adocicada revelou que a concentração de sacarose variou nos diferentes estudos. A revisão realizada por Willner (1997) indica que a concentração de sacarose a $2 \%$ produziu um padrão estável de ingestão desse líquido na linha de base. Willner (1997) afirma que a curva de consumo de líquidos em roedores tem a forma de $\mathrm{U}$ invertido em relação à concentração de sacarose com o maior consumo nos valores intermediários de concentração, salientando que as variáveis que explicariam este fato não estão estabelecidas.

No que diz respeito aos testes de consumo, alguns estudos (Dolabela, 2004; Oliveira, 2009; Thomaz, 2001, 2009) que adotaram a concentração de $2 \%$ de sacarose observaram que o consumo de água adocicada diminuiu entre $10 \mathrm{~g}$ e $25 \mathrm{~g}$ após a submissão ao PE. Entretanto, em outros estudos que utilizaram essa mesma concentração (Almeida, 2013; Fonseca Júnior et al., 2015; Lacerda, 2013; Pereira \& Sério, 2010; Rocha 2013) não foi possível observar esse decréscimo nos testes de consumo. Com a concentração de $8 \%$ de sacarose, alguns estudos (Dolabela, 2004; Rodrigues, 2005; Thomaz, 2001) que avaliaram a taxa de respostas de pressão à barra sob um esquema Conc FRFR - uma barra associada com água e a outra com água adocicada observaram uma diminuição na taxa de respostas no componente associado com água adocicada após à submissão ao PE. Entretanto, empregando um esquema de reforçamento diferente (Conc VIVI), Cardoso e Banaco (2009) não obtiveram os mesmos dados, o que sugere que o esquema de reforçamento pode ter sido uma das variáveis responsáveis pela diferença de resultados.

Segato, Castro-Souza, Segato, Morato e Coimbra (1997) e Pereira e Sério (2010) apresentaram a hipótese de que a quantidade de testes de consumo de água e de água com sacarose poderia ser uma variável importante na determinação da diminuição da preferência por água com sacarose, sugerindo que a exposição prolongada à água adocicada produziria uma analgesia nos animais, atenuando os efeitos dos estímulos aversivos presentes no PE. Entretanto, a redução na preferência por água com sacarose após a submissão ao PE foi observada tanto em estudos que utilizaram poucos testes - três testes (Oliveira, 2009) e quatro testes (Cardoso \& Banaco, 2009; Thomaz, 2001, 2009) quanto em estudos que empregaram uma quantidade maior de testes (12 e 16 nos estudos de Dolabela, 2004 e Rodrigues, 2005, respectivamente) antes do PE. Por outro lado, Almeida (2013), Fonseca Júnior et al. (2015), Lacerda (2013) e Rocha (2013) - que empregaram quatro testes - assim como Pereira e Sério (2010) - que realizaram 12 testes antes do PE - não observaram um decréscimo na preferência por água com sacarose nos testes de consumo. Apesar da variação na concentração de sacarose e na quantidade de testes, a descrição de como os testes foram realizados por esses autores não nos permite supor que tenha havido alguma outra diferença na forma como esses foram realizados.

As pesquisas que investigaram os efeitos do PE sobre a preferência de água com sacarose têm avaliado também outras variáveis como o peso dos animais ao longo dos estudos (Almeida, 2013; Cardoso \& Banaco, 2009; Dolabela, 2004; Fonseca Júnior et al., 2015; Lacerda, 2013; Oliveira, 2009; Pereira \& Sério, 2010; Rocha, 2013; Rodrigues, 2005; Thomaz, 2001, 2009) e quase todas observaram que os sujeitos diminuíram seus pesos após a submissão ao PE. Essa redução nos pesos corporais não foi observada por Pereira e Sério (2010) que retiraram o estressor "privação de ração" do protocolo para um subgrupo. Vale ressaltar que a privação de água e/ou de ração tem, por si só, um efeito sobre o peso dos animais e sobre o consumo de água e de ração na gaiola viveiro e isto pode ter mascarado os efeitos do PE sobre essas medidas.

O tipo de privação adotado parece ser também uma variável relevante nos dados obtidos nessa área de investigação, quer seja ela parte integrante do PE (i.e., privação de água e/ou de ração enquanto estímulos estressores) quer seja ela uma variável cujo efeito afetará a motivação para o responder nas sessões operantes. Nos 
diversos estudos citados os animais foram submetidos a diferentes tipos de privação de água e de ração antes dos testes de consumo e das sessões operantes. Barr e Phillips (1998) adotaram uma privação de 20 horas de água e restringiram o acesso dos sujeitos à ração na gaiola viveiro, disponibilizando apenas $45 \mathrm{mg}$ de ração diariamente ao longo de todo o estudo, a fim de manter os animais a $90 \%$ dos seus pesos ad lib. Esses autores não observaram uma redução na preferência por água com sacarose após à submissão ao PE.

Após privarem seus animais de alimento por seis ou 23 horas antes das sessões operantes, Willner et al. (1998) tampouco observaram um decréscimo na preferência pela barra associada à água com sacarose em vigência do PE. Thomaz (2001), Dolabela (2004) e Rodrigues (2005) colocaram seus animais num regime de privação de água para mantê-los a $85 \%$ dos seus pesos ad lib, além de privá-los de água e de ração por 23 horas antes dos testes de consumo e observaram que houve uma redução de $10 \mathrm{~g}$ a $25 \mathrm{~g}$ no consumo de água com sacarose nos testes de consumo. No entanto, com o mesmo esquema de privação, Almeida (2013), Lacerda (2013) e Rocha (2013) obtiveram um aumento médio de $5 \mathrm{~g}$ a $20 \mathrm{~g}$ no consumo de água com sacarose após a submissão ao PE.

Já Cardoso e Banaco (2009) empregando 23,5 horas de privação de água diariamente mais privação de ração por 23 horas antes dos testes de consumo, Pereira e Sério (2010) utilizando 23 horas de privação de água e de ração antes dos testes de consumo e Fonseca Júnior et al. (2015) submetendo seus sujeitos a 48 horas de privação de água e de ração antes dos testes de consumo e das sessões operantes tampouco observaram um decréscimo na preferência na água adocicada. Diferentemente, Thomaz (2009), utilizando uma privação de 23 horas de água e de ração apenas antes dos testes de consumo, observou um decréscimo de 15 g a 25 g no consumo de água com sacarose durante o PE.

Uma meta-análise recente (Antoniuk, Ponimaskin, \& Wlodarczyk, 2019) apontou que a maioria dos trabalhos internacionais nessa área tem se preocupado em avaliar a resposta consumatória (i.e., consumo de água com sacarose) envolvidas no modelo de anedonia, diferentemente do foco do modelo operante (i.e., resposta instrumental) adotado no presente estudo e considerado por Willner et al. (1998) uma medida alternativa como índice "confiável" de responsividade à recompensa.

Considerando a divergência de resultados nos diferentes estudos com diferentes tipos de privação, o presente estudo teve como objetivos verificar os efeitos do PE na escolha entre água pura e água com sacarose numa concentração de $2 \%$ em testes de consumo, na frequência de respostas em duas barras - uma barra associada com água com sacarose e outra com água pura - sob um esquema Conc VIVI em ratos sob um regime de privação apenas de água de modo a mantê-los a $85 \%$ dos seus pesos ad lib.

\section{MÉTODO}

O trabalho foi aprovado pelo CEUA (Comitê de Ética e no Uso de Animais da Pontifícia Universidade Católica de São Paulo). Número do processo: 0011/15.

\section{Sujeitos}

Foram utilizados 11 ratos machos, experimentalmente ingênuos, com aproximadamente 90 dias de idade no início do experimento, da linhagem Wistar, provenientes do biotério da Santa Casa de Misericórdia de São Paulo.

\section{Ambiente, Materiais e Equipamentos}

Duas salas do biotério com dimensões aproximadas de $0,90 \mathrm{~m}$ de largura $x \quad 2,0 \mathrm{~m}$ de profundidade $\mathrm{x} 2,80 \mathrm{~m}$ de altura serviram de ambiente para (1) a aplicação do PE e o (2) alojamento do sujeitocontrole que não foi submetido ao PE. Os animais foram alojados individualmente em gaiolas metálicas de $20 \mathrm{~cm}$ (Largura) x $30 \mathrm{~cm}$ (Profundidade) X $21 \mathrm{~cm}$ (Altura), permanecendo nessa condição até o término do experimento, exceto os sujeitos submetidos ao estressor "agrupamento", descritos detalhadamente na seção PE. $O$ ciclo de 12 horas claro e 12 horas de escuro foi regulado por um timer automático (exceto durante um período especifico durante a submissão ao PE no qual houve iluminação contínua). A temperatura e a umidade foram aferidas diariamente, por meio de um termohigrômetro em cada sala. A temperatura média da sala variou entre 20 a $23^{\circ} \mathrm{C}$ com umidade relativa do ar entre 40 a $60 \%$.

Foram utilizados: a) uma balança digital com precisão de $0,5 \mathrm{~g}$ para aferição do peso e do alimento dos sujeitos, b) 31 garrafas de plástico com capacidade para $250 \mathrm{ml}$ de líquido. Dessas, 20 garrafas foram usadas nos testes semanais de consumo nos quais eram oferecidas simultaneamente água e água com sacarose a 2\%, uma em cada garrafa, e as demais foram usadas para a disponibilização diária de água, c) seringas descartáveis para medir as quantidades disponibilizadas e consumidas de água e de água com sacarose, d) um Luxímetro: modelo MLM-1011-Minipa para garantir a mesma intensidade de luz em ambas as salas utilizadas; e) um Decibelímetro: modelo 407727-Extech a fim de garantir que o estressor barulho tivesse a intensidade máxima de 85 decibéis, f) Caixas viveiro feitas de material plástico e dimensões $30 \mathrm{~cm}$ (Largura) x $45 \mathrm{~cm}$ (Profundidade) x 16 $\mathrm{cm}$ (Altura), g) 11 Gaiolas viveiro já descritas no item Ambiente, h) Sacarose da marca Merck, i) Quatro caixas de condicionamento operante MedAssociates ${ }^{\circledR}$, modelo Env-008 com duas barras, posicionadas frente a frente na lateral direita e na lateral esquerda, a $5 \mathrm{~cm}$ da base da gaiola, pressionáveis mediante força de $15 \mathrm{~N}$. Cada barra podia, por meio de um circuito eletromecânico, acionar um mecanismo que liberava os líquidos utilizados como reforçadores (água e água com sacarose). A apresentação dos estímulos e o registro das respostas dos sujeitos foram controlados pelo software Schedule Manager para Windows produzido pela Med Associates ${ }^{\circledR}$. 


\section{Procedimento}

Com três meses de vida, os animais foram alojados em gaiolas individuais e a partir de então foram pesados diariamente. Os sujeitos foram nomeados de S1 a S11.

\section{líquidos.}

Testes de consumo e de preferência de

Testes com uma garrafa. Quando os sujeitos completaram 148 dias de vida foi realizado o primeiro teste de consumo de água com sacarose. Este consistiu em apresentar aos sujeitos apenas uma garrafa contendo $100 \mathrm{ml}$ de água com sacarose numa concentração a $2 \%$ durante um período de uma hora na gaiola viveiro. $\mathrm{O}$ consumo do líquido foi calculado pela diferença entre a quantidade disponibilizada e a sobra do líquido ao término do período de uma hora.

Sete dias após o primeiro teste, foi realizado um segundo teste idêntico ao primeiro, exceto em relação ao lado da gaiola em que a garrafa foi colocada.

Testes com duas garrafas. Sete dias após o segundo teste com uma garrafa, foi realizado o primeiro teste com duas garrafas, uma contendo $100 \mathrm{ml}$ de água e a outra $100 \mathrm{ml}$ de água com sacarose numa concentração a $2 \%$ com o objetivo de avaliar a preferência dos animais. Os testes com duas garrafas foram realizados uma vez por semana até o final do experimento. Assim como os testes anteriores, cada teste durava uma hora e era realizado sempre nos mesmos dias e horários e as garrafas foram disponibilizadas na gaiola viveiro de forma simultânea (duas garrafas disponibilizadas ao mesmo tempo), equidistantes das laterais da caixa tendo os lados de apresentação das garrafas sido alternados semanalmente.

$P E$. Empregou-se o mesmo protocolo descrito por Almeida (2013), Dolabela (2004), Lacerda (2013), Oliveira (2009), Pereira e Sério (2010) Rocha (2013), Rodrigues (2005) e Thomaz (2001, 2009), exceto pela exclusão dos estressores "privação de ração", "acesso restrito à comida" e "privação de água". Nem todos os estressores foram apresentados ao mesmo tempo. Cada sujeito foi submetido a um ou dois componentes do protocolo em todos os dias da semana, durante seis semanas. Os estressores componentes do protocolo no presente estudo foram: a) uma luz estroboscópica, localizada no chão da sala, ligada por 2 ou 7 horas disparando 300 flashes por minuto, b) inclinação da gaiola viveiro em $30^{\circ}$ para trás, permanecendo assim por 7 ou 17 horas, c) maravalha molhada com $50 \mathrm{ml} \mathrm{de}$ água colocada no chão da gaiola viveiro por 17 horas tornando-a suja, d) um ruído branco intermitente de 85 decibéis ligado por 2 ou 5 horas na sala onde foi aplicado o PE, e) um cheiro produzido por um desodorante purificador de ar com injeção de flagrância temporizada (a cada 15 minutos durante 17 horas) na sala onde foi aplicado o PE, f) agrupamento: dois sujeitos foram colocados em uma mesma gaiola viveiro, tanto na gaiola original de um deles quanto na do outro por 17 horas, g) um objeto de metal redondo de $7 \mathrm{~cm}$ de diâmetro e $1,5 \mathrm{~cm}$ de altura foi colocado na gaiola viveiro e deixado por um período de 17 horas e h) a luz da sala onde foi aplicado o PE permaneceu acesa por 19 horas seguidas. A Figura 1 apresenta a distribuição dos estressores ao longo da semana. O protocolo durou seis semanas, conforme proposto por Willner et al. (1987).

Condições experimentais. Quando os animais completaram 152 dias de vida, foram distribuídos aleatoriamente em cinco condições. A Figura 2 apresenta a distribuição dos sujeitos pelas condições experimentais.

Oito sujeitos (S1, S2, S4, S6, S8, S9, S10 e $\mathrm{S} 11)$ foram submetidos aos testes de consumo de água e de água com sacarose numa concentração a $2 \%$, ao PE e às sessões operantes. $\mathrm{O}$ procedimento de automodelagem da resposta de pressão à barra ocorreu para esses animais quando completaram 152 dias de vida. Houve instalação da resposta de pressão à barra da direita e, no dia seguinte, instalação da resposta de pressão à barra da esquerda, ambas mantidas por reforçamento com água $(0,1 \mathrm{ml}$ para cada resposta) em esquema de reforçamento contínuo (CRF) até que fossem apresentadas 100 respostas de pressão à barra ou transcorridos 50 minutos, o que ocorresse primeiro.

Posteriormente, esses animais foram submetidos a um esquema de reforçamento ConcVI5segVI5seg. Os intervalos do esquema de intervalo variável VI5seg foram: 7,9 - 5,1 - 5,3 - 6,5$4,2-3,5-9,1-3,2-1,7$ e 3,5 gerados automaticamente, conforme proposto por Pessôa e Buffara (2005).

Num dos componentes do esquema concorrente o reforço era água pura e no outro água adocicada com sacarose numa concentração de $8 \%$, como descrito por Cardoso e Banaco (2009), Dolabela (2004), Rodrigues (2005) e Thomaz (2001). O grupo denominado "Antes e Depois" (AD) - composto pelos sujeitos S1, S4, S9 e S11 - foi submetido a 21 sessões nas quais estava em vigor o ConcVI5seg (água)VI5seg(água+8\%sac), das quais 12 sessões precederam a apresentação do PE e nove a sucederam. O grupo denominado "Antes, Durante e Depois" (ADD) - composto pelos sujeitos S2, S6, S8 e S10 - foi submetido a 27 sessões nas quais estava em vigor o esquema ConcVI5seg (água)VI5seg(água+8\%sac), das quais 12 sessões precederam a apresentação do $\mathrm{PE}$, seis ocorreram em vigência do protocolo e nove após a suspensão do mesmo. 


\begin{tabular}{|c|c|c|c|c|c|c|c|}
\hline Horas & Domingo & Segunda & Terça & Quarta & Quinta & Sexta & Sábado \\
\hline $00: 00$ & \multirow{11}{*}{ Agrupamento } & \multirow{11}{*}{ Cheiro } & \multirow{8}{*}{$\begin{array}{c}\text { Iluminação } \\
\text { contínua }\end{array}$} & \multirow{11}{*}{ Inclinaç̆o da gaiola } & \multirow{11}{*}{ Gaiola suja } & \multirow{8}{*}{$\begin{array}{l}\text { Iluminação } \\
\text { continua }\end{array}$} & \multirow{11}{*}{ Objeto estranho } \\
\hline $01: 00$ & & & & & & & \\
\hline $02: 00$ & & & & & & & \\
\hline $03: 00$ & & & & & & & \\
\hline 04:00 & & & & & & & \\
\hline 05:00 & & & & & & & \\
\hline $06: 00$ & & & & & & & \\
\hline $07: 00$ & & & & & & & \\
\hline $08: 00$ & & & & & & & \\
\hline 09:00 & & & & & & & \\
\hline 10:00 & & & & & & & \\
\hline 11:00 & & & Teste de consumo & \multirow{2}{*}{ Luz estroboscópica } & \multirow[t]{2}{*}{ Sessão operante } & \multirow{7}{*}{ Luz estroboscópica } & \multirow{7}{*}{ Inclinação da gaiola } \\
\hline 12:00 & & & & & & & \\
\hline $13: 00$ & \multirow{5}{*}{$\begin{array}{c}\text { Barulho } \\
\text { intermitente }\end{array}$} & & & & & & \\
\hline 14:00 & & & & & & & \\
\hline $15: 00$ & & & & & \multirow{3}{*}{$\begin{array}{l}\text { Barulho } \\
\text { intermitente }\end{array}$} & & \\
\hline $16: 00$ & & & & & & & \\
\hline $17: 00$ & & & & & & & \\
\hline $18: 00$ & \multirow{6}{*}{ Cheiro } & & \multirow{6}{*}{ Inclinaç̧ăo da gaiola } & \multirow[t]{6}{*}{ Gaiola suja } & & \multirow{6}{*}{ Objeto estranho } & \multirow{6}{*}{ Agrupamento } \\
\hline 19:00 & & & & & & & \\
\hline $20: 00$ & & \multirow{4}{*}{$\begin{array}{l}\text { Iluminarẵa } \\
\text { continua }\end{array}$} & & & \multirow{4}{*}{$\begin{array}{l}\text { lluminaçăo } \\
\text { continua }\end{array}$} & & \\
\hline 21:00 & & & & & & & \\
\hline 22:00 & & & & & & & \\
\hline 23:00 & & & & & & & \\
\hline
\end{tabular}

Figura 1. Distribuição semanal dos estímulos utilizados no PE.

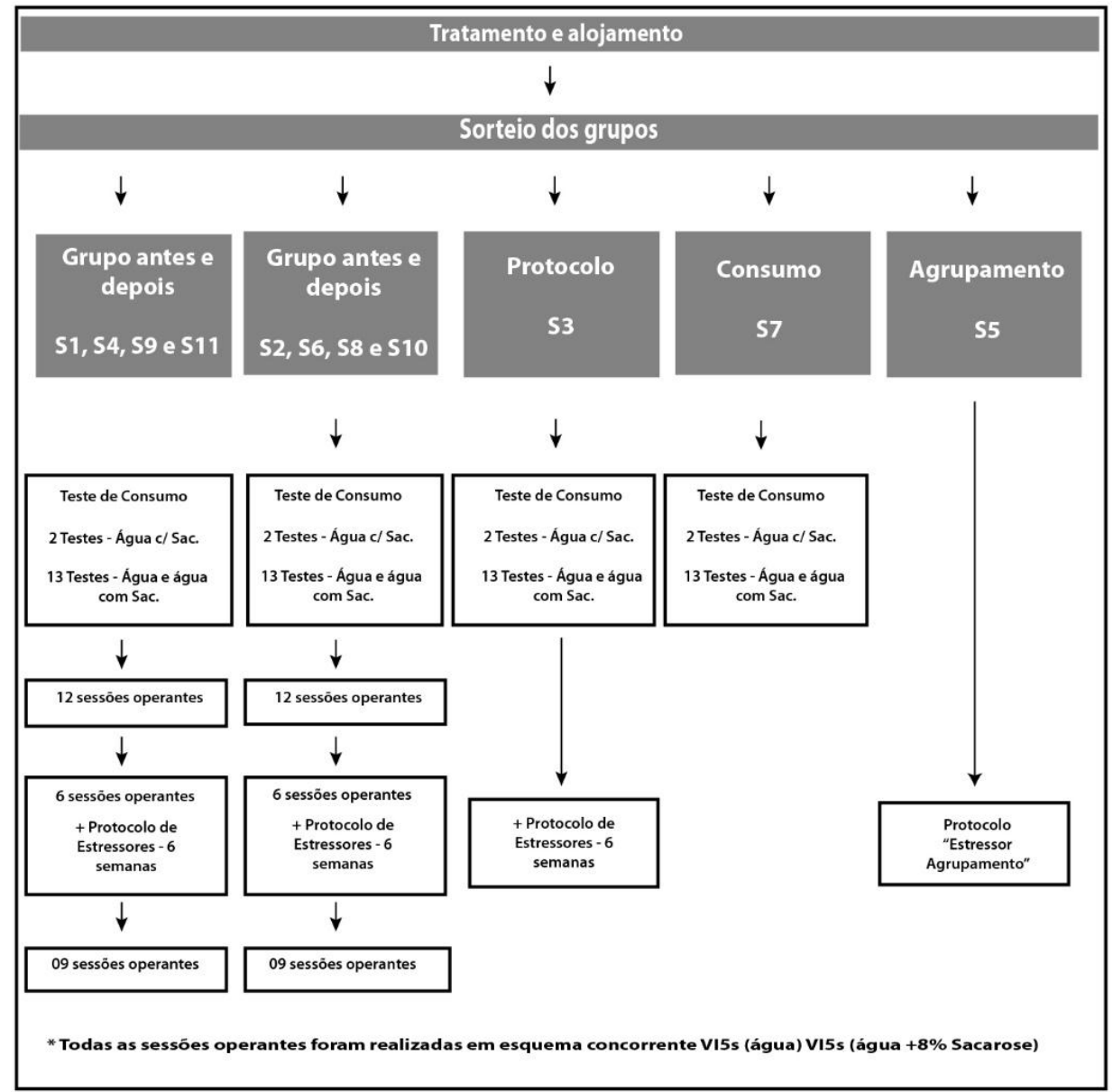

Figura 2. Distribuição dos sujeitos nas diferentes condições experimentais. 
Para esses dois grupos (AD e ADD) as sessões operantes ocorreram quatro vezes por semana no mesmo período do dia e terminavam após a liberação de 100 reforços ou após 20 minutos, o que ocorresse primeiro. Nas primeiras sete sessões, se o sujeito apresentasse pelo menos uma resposta a mais numa das barras por duas sessões consecutivas, a água e a água com sacarose eram alternadas nos bebedouros esquerdo e direito da caixa experimental da sessão seguinte. A partir da $8^{a}$ sessão, a alternância dos líquidos ocorreu após cada sessão, caso o animal apresentasse uma preferência por uma das duas barras. $\mathrm{O}$ animal S5 foi utilizado apenas para possibilitar a ocorrência do estressor "agrupamento". O sujeito S7 foi submetido apenas aos testes de consumo de água e de água com sacarose numa concentração a $2 \%$ e o sujeito $\mathrm{S} 3$ (Protocolo) foi submetido aos testes de consumo e ao PE conforme apresentado na Figura 2.

\section{RESULTADOS}

A Figura 3 mostra os pesos corporais de cada sujeito ao longo do estudo. Do primeiro ao $34^{\circ}$ dia (período que antecedia o início da privação), os animais apresentaram um ganho de peso. Com a introdução da privação de água que tinha por objetivo manter os animais a $85 \%$ dos seus pesos ad lib, todos os sujeitos atingiram os valores almejados, e como se pode ver, a submissão ao PE não foi acompanhada por uma diminuição nos pesos dos sujeitos.
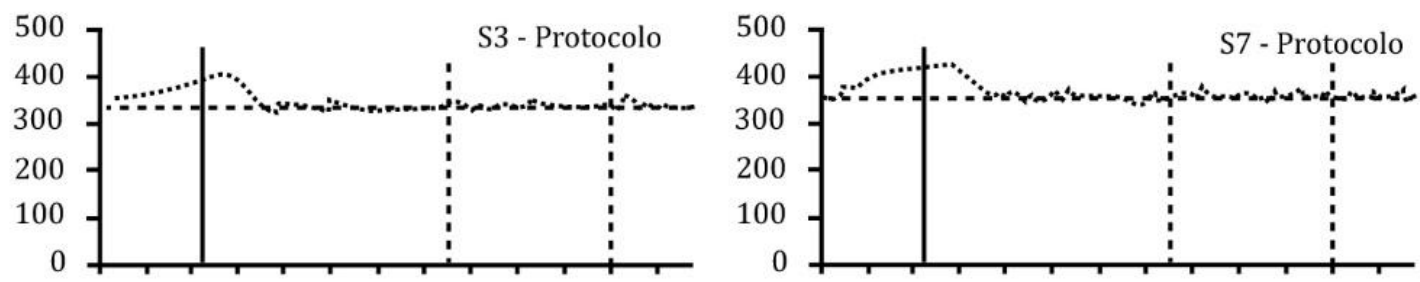

(Grupo Antes e Depois)
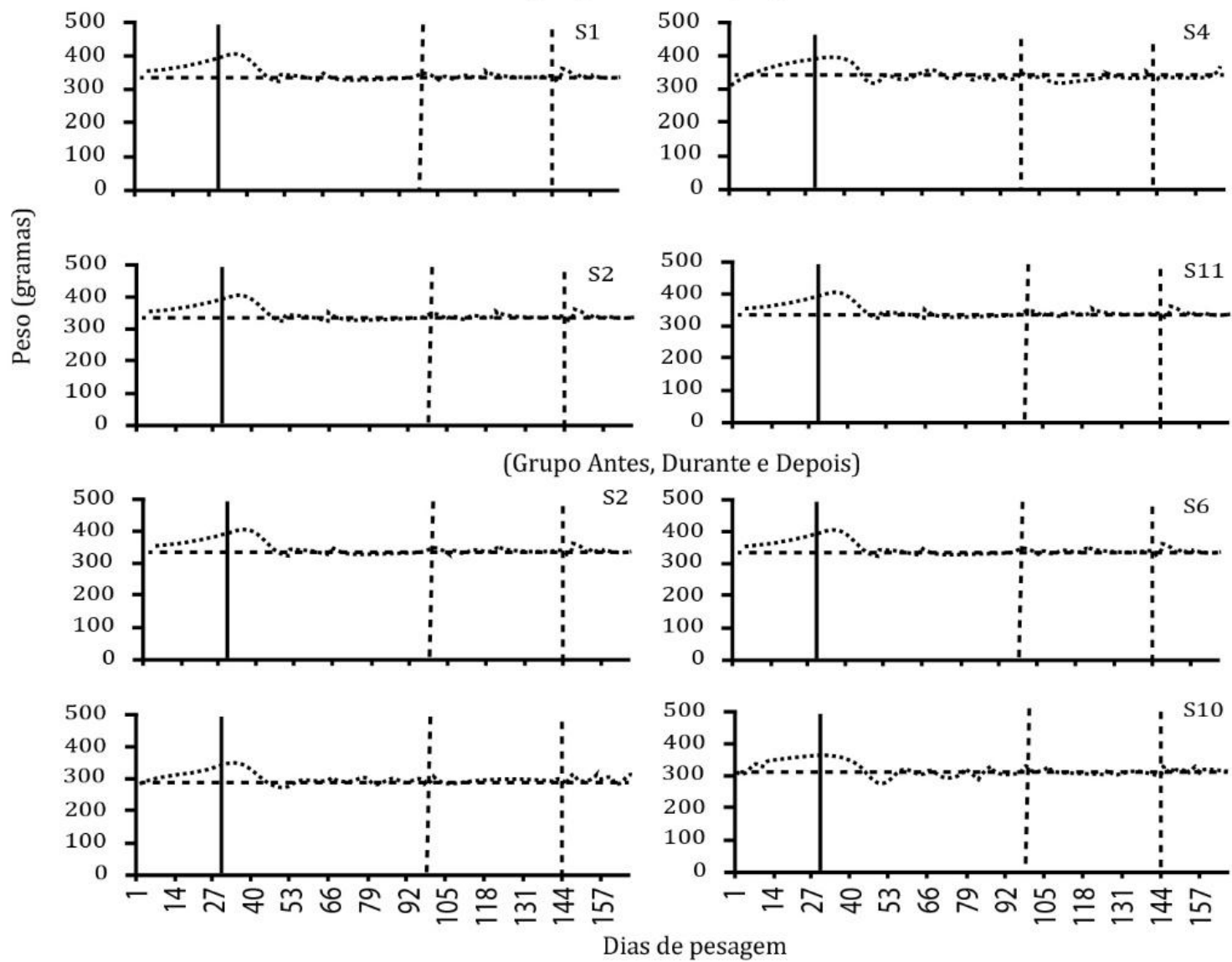

Figura 3. Peso corporal dos sujeitos S1, S4, S9 e S11 (Grupo AD), S2, S6, S8, S10 (Grupo ADD), S3 Protocolo e S7 Controle. A linha vertical inteira representa o início da restrição de água para atingir $85 \%$ dos pesos ad lib., a linha horizontal pontilhada representa o valor correspondente a $85 \%$ dos pesos ad lib. dos sujeitos e as duas linhas verticais pontilhadas indicam, respectivamente, o início e o fim do PE Estressores. 
A Figura 4 mostra o consumo de água e de água com concentração de sacarose a $2 \%$ pelos animais nos 13 testes semanais com duas garrafas. O sujeito S7, que foi submetido apenas aos 13 testes de consumo (sem passar pelo PE), apresentou uma preferência por água com sacarose em todos os testes de consumo. Os quatro primeiros testes foram marcados por um alto consumo de água com sacarose, chegando a $40 \mathrm{ml}$

no $2^{\circ}$ e no $4^{\circ}$ testes, porém a diferença entre o consumo de água adocicada e de água pura diminuiu para 31, 25 e 23,5 $\mathrm{ml}$ nos testes $5^{\circ}, 6^{\circ}$ e $7^{\circ}$, respectivamente. Percebeu-se que após o quarto teste de consumo houve um problema técnico no temporizador automático do ciclo claro/escuro, localizado na sala em que esse animal foi alojado. Após a solução desse problema técnico no $8^{\circ}$ teste, o consumo de água com sacarose aumentou nos testes subsequentes, variando entre $24 \mathrm{ml}$ no $8^{\circ}$ teste e $32 \mathrm{ml}$ no $12^{\circ}$ teste, sem atingir os níveis semelhantes àqueles observados nos quatro primeiros testes. O sujeito S3 (Protocolo) que foi submetido aos testes de consumo e ao $\mathrm{PE}$, mas não às sessões operantes, apresentou uma preferência por água com sacarose nos quatro primeiros testes (antes da submissão ao $\mathrm{PE}$ ) atingindo o valor máximo de consumo de $30 \mathrm{ml}$ no $1^{\circ}$ teste. Após a introdução do PE (que ocorreu do $5^{\circ}$ ao $10^{\circ}$ teste), houve uma oscilação na preferência entre os dois líquidos até o final do estudo.
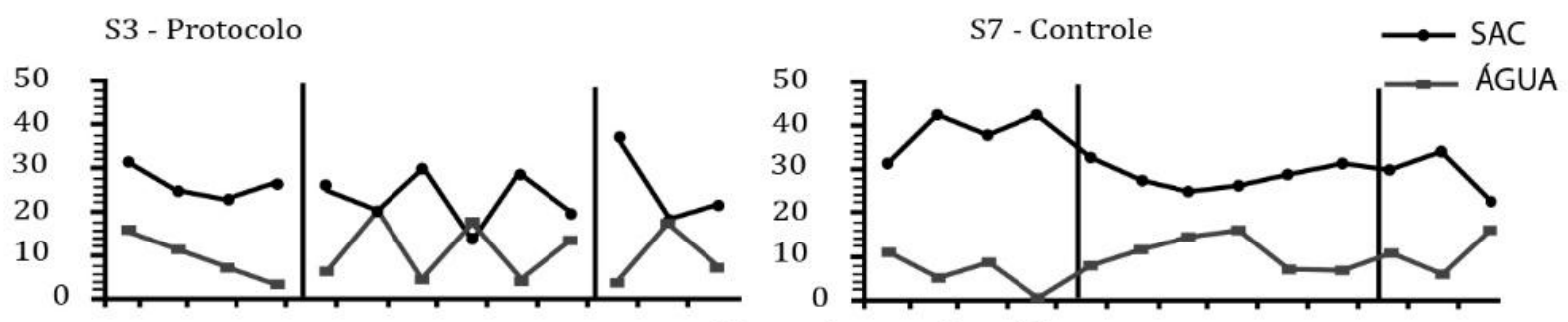

(Grupo Antes e Depois)
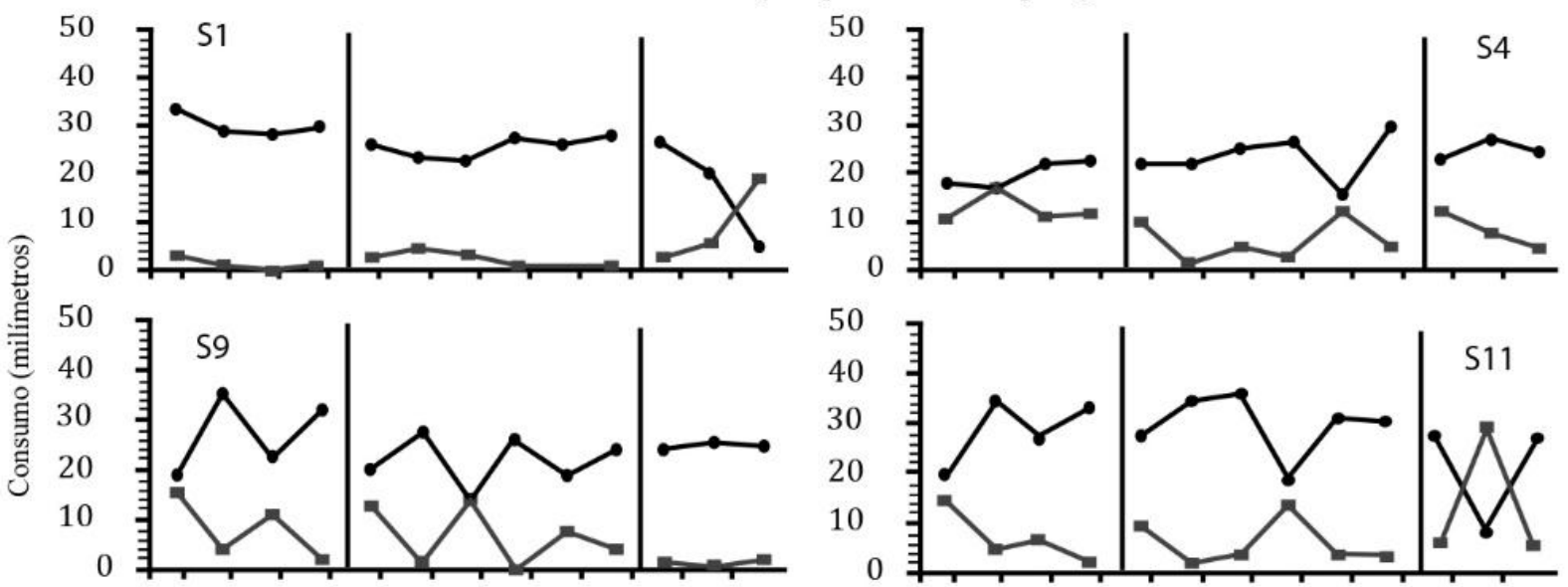

(Grupo Antes, Durante e Depois)
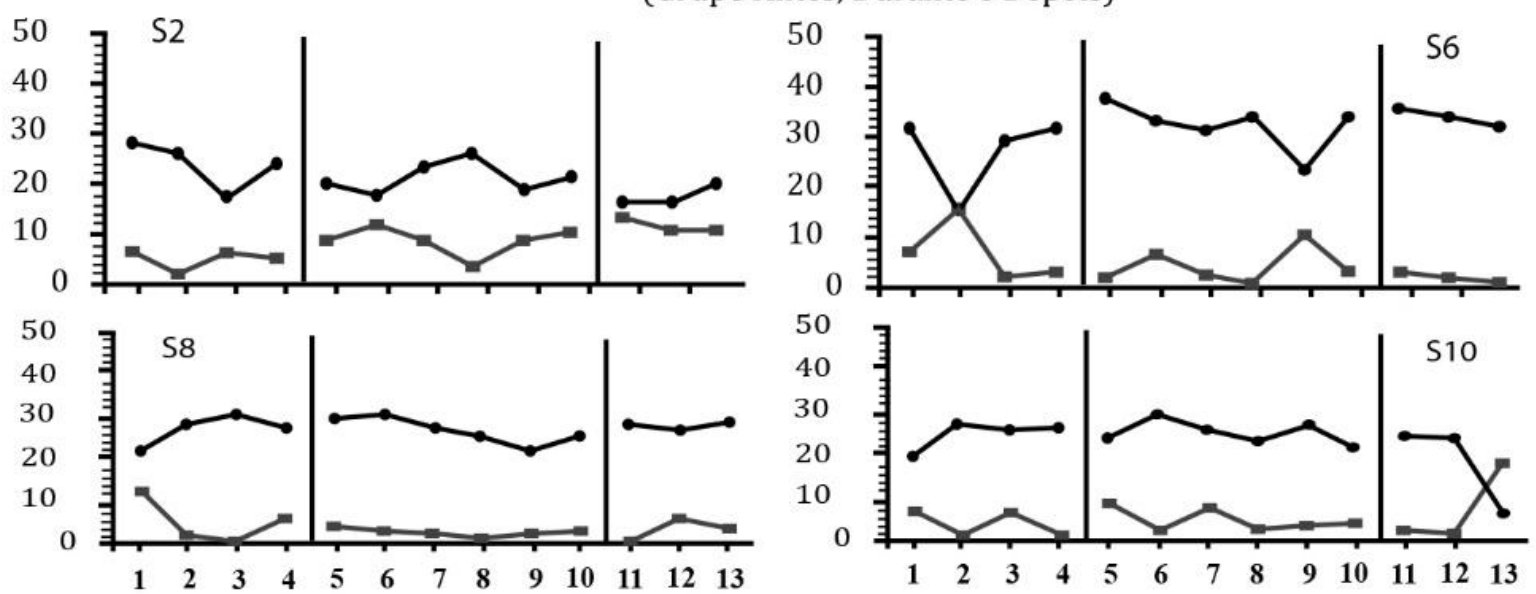

Semanas

Figura 4. Consumo de água e de água com sacarose para os sujeitos S1, S4, S9 e S11 (Grupo AD), S2, S6, S8, S10 (Grupo ADD), S3 Protocolo e S7 Controle nos testes de consumo semanais. O espaço entre as linhas verticais corresponde ao período de exposição ao PE Estressores. 
Os sujeitos do grupo $\mathrm{AD}$ (S1, S4, S9 e S11) submetidos aos testes de consumo e às sessões operantes antes e depois do PE - também apresentaram uma preferência por água com sacarose nos testes de consumo antes da submissão ao PE.

A Figura 4 indica os valores de consumo de água e de água com sacarose antes, durante e depois do PE para os sujeitos do grupo AD. A média de consumo de água com sacarose para esses sujeitos antes da submissão foi 26,67 g (DP 4,55); durante a submissão a média foi 25,15 g (DP 3,54) e após a submissão a média foi 22,15 g (DP 3,78 ). O teste de Friedman mostrou que essa diferença não atingiu uma significância estatística $(p=0,78)$.

Os dados dos sujeitos do grupo ADD (S2, S6, S8 e S10) - submetidos aos testes de consumo e às sessões operantes antes, durante e depois do PE - são muito semelhantes aos dos sujeitos do grupo AD. A média de consumo de água com sacarose para esses sujeitos antes da submissão foi $25,85 \mathrm{~g}$ (DP 1,38); durante a submissão a média foi $26,47 \mathrm{~g}$ (DP 4,24) e após a submissão a média foi $24,47 \mathrm{~g}$ (DP 7,72). O teste de Friedman mostrou que essa diferença não atingiu uma significância estatística ( $p$ $=1,00)$.

A Figura 5 mostra a frequência de respostas à barra associada com água e à barra associada com água com sacarose a $8 \%$ sessões operantes. Como se pode ver, antes da submissão ao PE todos sujeitos do grupo AD (S1, S4, S9 e S11) e um sujeito (S2) do Grupo ADD oscilaram na escolha das diferentes barras, isto é, ora escolhiam a barra associada com água, ora escolhiam a barra associada com água com sacarose. Sob o PE, os sujeitos do grupo ADD apresentaram um padrão muito semelhante ao da fase anterior (antes da submissão ao PE). Depois de suspenso o PE, os animais continuaram apresentando os mesmos padrões de escolha das fases anteriores.

(Grupo Antes e Depois)
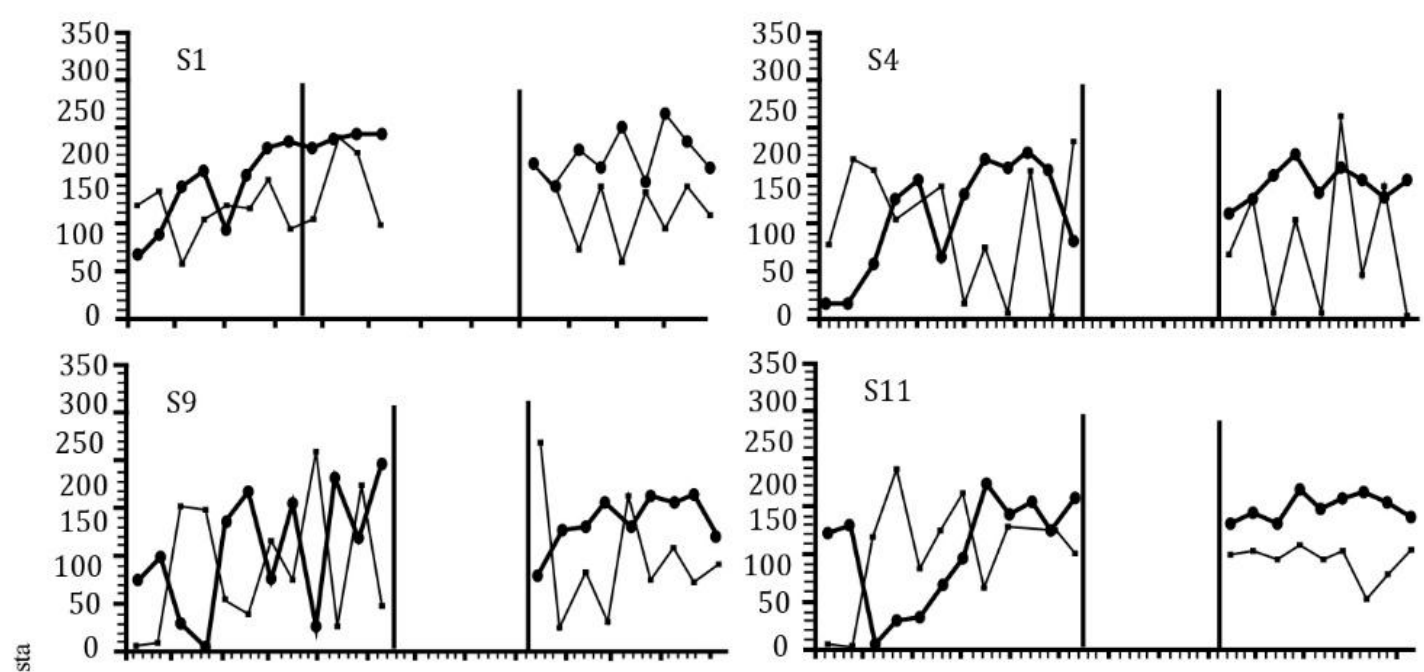

(Grupo Antes, Durante e Depois)
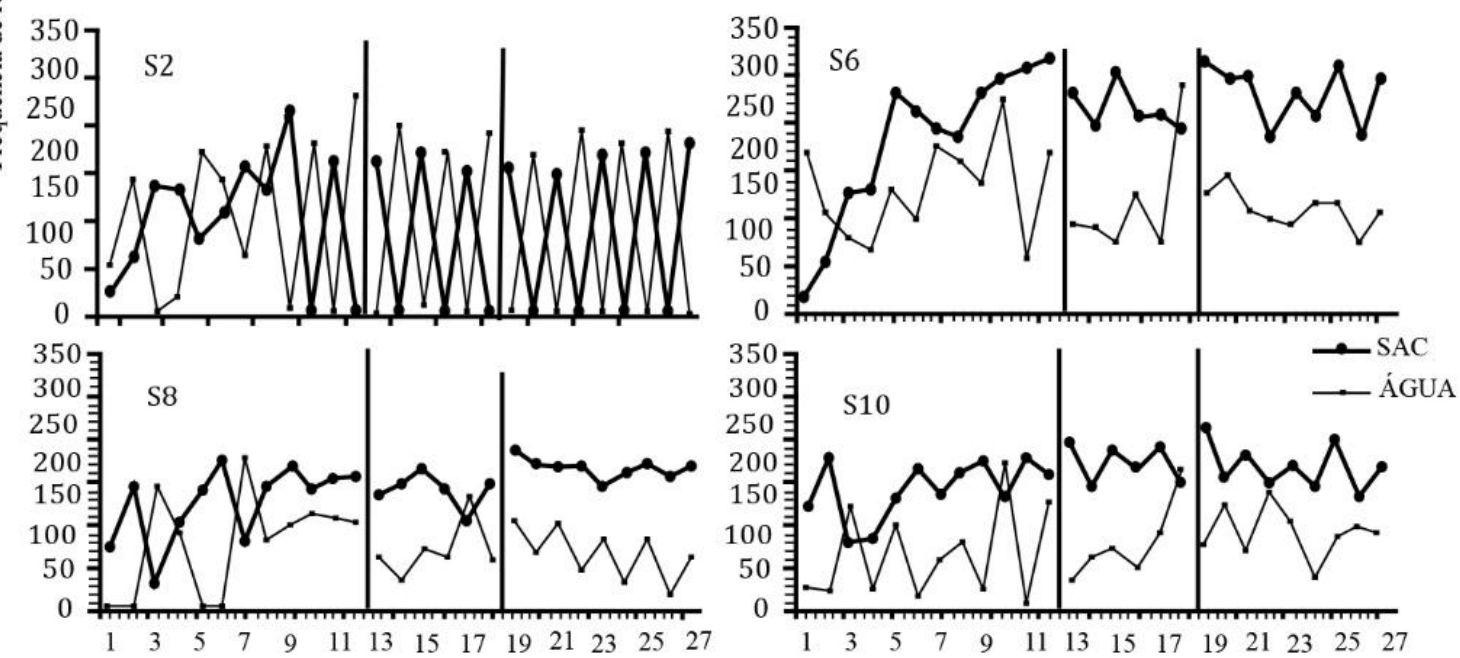

Sessões

Figura 5. Frequência de respostas apresentadas pelos sujeitos S1, S4, S9 e S11 (Grupo AD) e S2, S6, S8, S10 (Grupo ADD) nas sessões operantes sob o esquema Conc VI5VI5. O espaço entre as linhas verticais corresponde ao período de exposição ao PE. 
Com o objetivo de verificar se o PE foi acompanhado por alguma alteração no consumo de ração na gaiola viveiro ao longo das 13 semanas em que ocorreram os testes de consumo de água e de água com sacarose, construiuse a Figura 6. Como se pode ver, na primeira semana em que o PE foi aplicado, houve um decréscimo no consumo de ração na gaiola viveiro para todos os animais submetidos a esse procedimento. Com a suspensão do PE, houve um retorno aos valores observados na linha de base.

Os dados dos sujeitos do grupo AD (S1, S4, S9 e S11) mostram que a média de consumo de ração na gaiola viveiro antes da submissão ao PE foi $21,5 \mathrm{~g}$ ( $\mathrm{DP}=2,98)$, durante 19,64 g ( DP = 1,92), e depois 20,87 ( $\mathrm{DP}=1,75)$. O teste de Friedman mostrou que essa diferença foi significativa $(p=0,039)$. Os dados dos sujeitos do grupo ADD (S2, S6, S8 e S10) mostram que a média de consumo de ração na gaiola viveiro antes da submissão ao PE foi 20,44 g (DP = 0,76), durante 17,81 g (DP = 1,42), e depois 21,08 (DP = 1,01). O teste de Friedman mostrou que essa diferença foi significativa $(p=0,039)$.

\section{DISCUSSÃO}

De um modo geral, os dados do presente estudo mostraram que a submissão ao PE não foi acompanhada por uma diminuição na preferência por água adocicada nos testes de consumo, replicando os dados de Almeida (2013), Fonseca Júnior et al., (2015), Lacerda (2013), Pereira e Sério (2010) e Rocha (2013). Também não foi observada uma redução na frequência de respostas durante as sessões operantes após a submissão ao PE, em concordância com os resultados apresentados por Almeida (2013), Barr e Phillips (1998), Cardoso e Banaco (2009), Fonseca Júnior et al. (2015), Oliveira (2009), Pereira e Sério (2010), Thomaz (2009) e Willner et al. (1998). A redução na preferência de 40 para $23,5 \mathrm{ml}$ por água adocicada nos testes de consumo por um único sujeito (S7) do $5^{\circ}$ ao $7^{\circ}$ testes talvez possa ser explicada pela maior exposição desse sujeito a um dos estímulos estressores do protocolo (luz contínua) decorrente de um problema técnico.
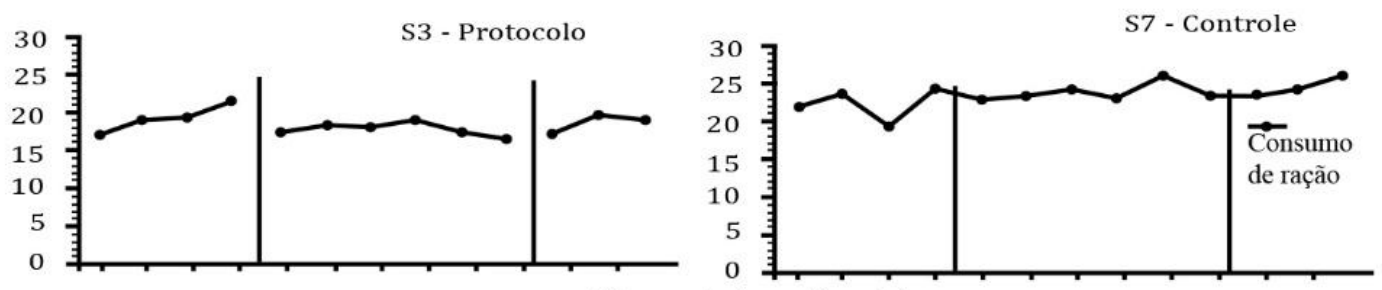

(Grupo Antes e Depois)
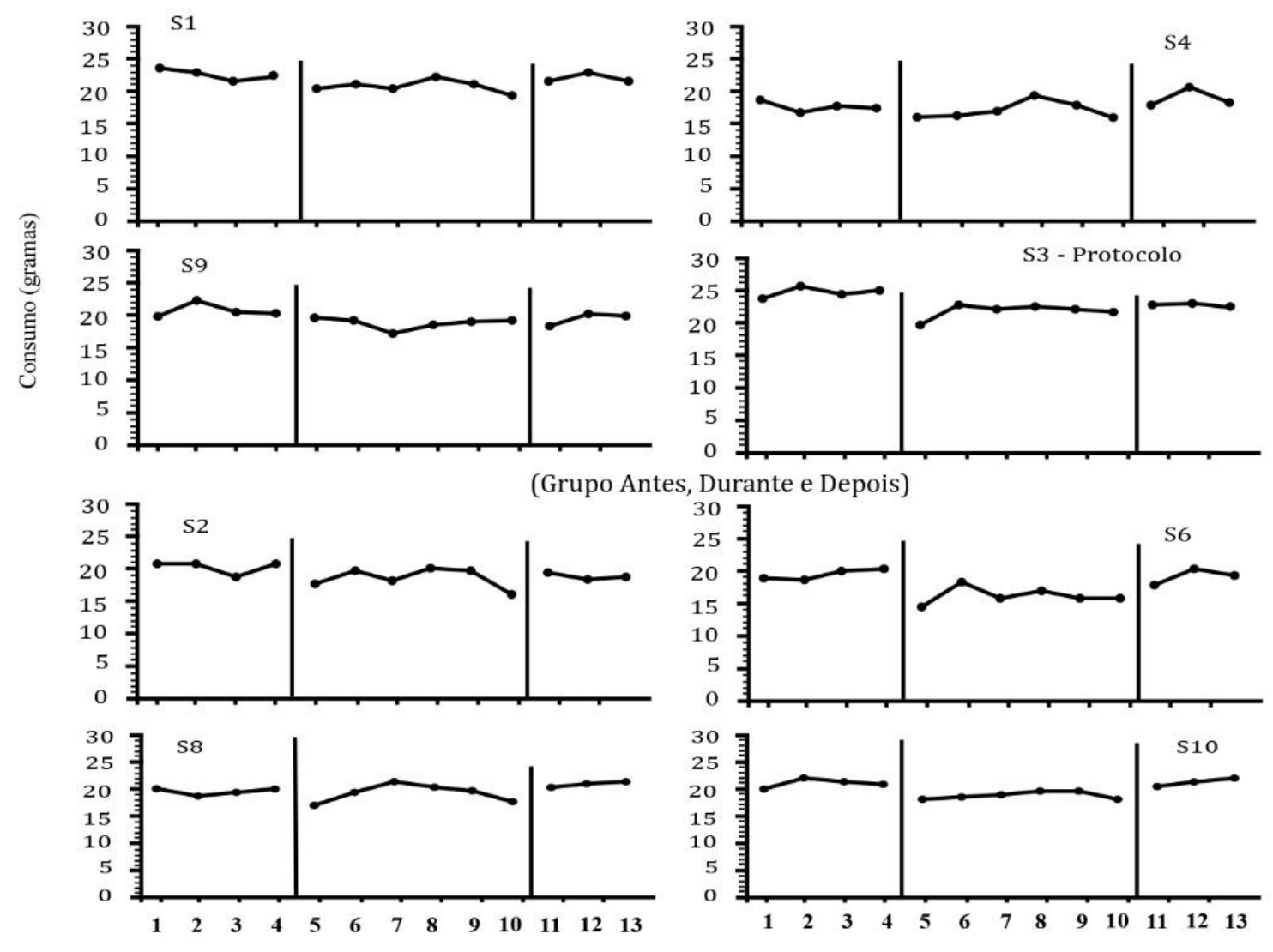

Semanas

Figura 6. Média semanal do consumo de ração na gaiola viveiro para cada sujeito ao longo das 13 semanas em que ocorreram os testes de consumo de água e de água com sacarose. O espaço entre as linhas verticais corresponde ao período de exposição ao PE. 
Diferentemente do que foi observado por Almeida (2013), Cardoso e Banaco (2009), Dolabela (2004), Fonseca Júnior et al. (2015), Lacerda (2013), Pereira e Sério (2010), Rocha (2013), Rodrigues (2005) e Thomaz (2001, 2009), no presente estudo a submissão ao PE não foi acompanhada por uma redução no peso dos animais. Os dados observados na presente pesquisa assemelham-se àqueles apresentados por quatro sujeitos de um subgrupo do estudo de Pereira e Sério (2010) submetidos ao PE sem a privação de ração, os quais tampouco apresentaram uma diminuição nos seus pesos. No presente estudo retirou-se o "estressor" privação de ração do $\mathrm{PE}$ assim como no subgrupo mencionado do estudo de Pereira e Sério (2010). É importante ressaltar que a disponibilidade de água ofertada aos animais durante o presente estudo pode também ter contribuído para a manutenção do peso dos animais, uma vez que o pesquisador deliberadamente alterava a oferta de água na gaiola viveiro de modo a manter os animais a $85 \%$ dos seus pesos ad lib.

Embora não tenha sido observada uma diminuição na preferência por água adocicada, os dados do presente estudo indicaram uma redução na quantidade de ração consumida na gaiola viveiro associada à submissão ao PE, semelhante àquela encontrada por Almeida (2013), Lacerda (2013) e Rocha (2013). Entretanto, vale ressaltar que naquelas pesquisas a "privação de ração" era um dos componentes do PE, ou seja, durante as seis semanas em que os animais foram submetidos ao PE, ocorreu uma privação de ração por períodos que variaram entre 17 e 44 horas semanais, diferentemente do presente estudo no qual a ração esteve sempre disponível antes, durante e depois da submissão ao PE.

A redução significativa no consumo de ração na gaiola viveiro observada no presente estudo paralelamente à submissão dos animais ao PE sugere que essa variável possa ser usada como uma medida alternativa à preferência por água com sacarose para avaliar os efeitos do PE sobre o valor reforçador de um estímulo. Diferentemente dos testes de consumo de água e de água com sacarose que geralmente ocorrem nos estudos dessa área, apenas durante uma hora uma vez por semana, a ração pode ficar continuamente disponível na gaiola viveiro, o que facilita a observação e o registro da sua ingestão.

Alguns autores (Pereira \& Sério, 2010; Willner et al, 1998) têm questionado o uso dos testes de preferência de água com sacarose e têm sugerido que se busquem medidas alternativas para avaliar o decréscimo no valor reforçador de um estímulo após a submissão ao PE - interpretado como anedonia. Os dados do presente estudo sugerem que o "consumo de ração na gaiola viveiro" pode ser uma dessas medidas. Entretanto, deve-se salientar que o presente estudo trabalhou com uma amostra reduzida o que constitui uma limitação para a generalidade dos resultados. Futuras replicações com um número maior de sujeitos poderiam confirmar se a redução no consumo de ração na gaiola viveiro seria um indicador de anedonia.

\section{DECLARAÇÃO DE CONFLITO DE INTERESSES}

Os autores declaram que não há conflito de interesses relativos à publicação do presente artigo.

\section{CONTRIBUIÇÃO DE CADA AUTOR}

Ambos os autores participaram igualmente da elaboração do trabalho de forma que tornam pública sua responsabilidade pelo conteúdo.

\section{DIREITOS AUTORAIS}

Este é um artigo aberto e pode ser reproduzido livremente, distribuído, transmitido ou modificado, por qualquer pessoa desde que usado sem fins comerciais. $\mathrm{O}$ trabalho é disponibilizado sob a licença Creative Commons 4.0 BY-NC.

\section{(cc) $\mathrm{BY}-\mathrm{NO}$}

\section{REFERÊNCIAS}

Antoniuk, S., Bijata, M., Ponimaskin, E., \& Wlodarczyk, J. (2019). Chronic Unpredictable Mild Stress for Modeling Depression in Rodents: Meta-analysis of Model Reliability. Neuroscience \& Biobehavioral Reviews, 99, 101-116. doi: 10.1016/j.neubiorev.2018.12.002

American Psychiatry Association (2013). Diagnostic and Statistical Manual of Mental disorders - DSM-5. 5th.ed. Washington: American Psychiatric Association.

Almeida, N. K. S. P. (2013). Chronic Mild Stress (CMS) e os efeitos da exposição de sujeitos a um esquema de reforçamento de tempo variável (Dissertação de Mestrado). Pontifícia Universidade Católica de São Paulo, São Paulo, Brasil.

Barr, A. M., \& Phillips, A. (1998). Chronic mild stress has no effect on responding by rats for sucrose under a progressive ratio schedule. Physiology and Behavior, 64, 591- 597. doi: 10.1016/S0031-9384(98)00060-2

Cardoso, L. R. D., \& Banaco, R. A. (2009). Efeitos do esquema de intervalo variável no desenvolvimento de anedonia induzida por estresse crônico moderado em ratos. Revista Brasileira de Análise do Comportamento, 5 (1) 79-96. doi: 10.18542/rebac.v5i1.723

Dolabela, A. C. F. O. (2004). Um estudo sobre as possíveis interações entre o Chronic Mild Stress e o desempenho operante (Dissertação de Mestrado). Pontifícia Universidade Católica de São Paulo, São Paulo, Brasil.

Fonseca Júnior, A. R., Castelli, M. C. Z., \& Oliveira, E. C. A. (2015). Effects of Chronic Mild Stress on Operant Discrimination Learning. Behavior Analysis: Research and Practice, 15, 20-27. doi: 10.1037/h0101066

Katz, R. J. (1982). Animal model of depression: Pharmacological sensitivity of a hedonic deficit. Pharmacology Biochemistry Behavior, 16, 965-968. doi: 10.1016/0091-3057(82)90053-3 
Lacerda, L. G. (2013). Chronic Mild Stress: Um estudo sobre interação entre manipulação neonatal $e$ submissão ao protocolo de estressores na vida adulta (Dissertação de Mestrado). Pontifícia Universidade Católica de São Paulo, São Paulo, Brasil.

Oliveira, A. C. F. (2009). Estresse Moderado Crônico: Efeitos sobre a atividade geral em ratos (Tese de Doutorado). Universidade de Brasília, Brasília - DF, Brasil.

Pereira, C. M., \& Sério, T. M. A. P. (2010). Chronic Mild Stress: Um Estudo Sobre a Interação entre submissão ao protocolo de estressores, comportamento operante e privação. Revista Brasileira de Análise do Comportamento, 6 (1), 67-88. doi: 10.18542/rebac.v6i1.994

Pereira, C. M. (2015). Efeito do protocolo de estressores e da privação de água sobre o consumo de sacarose, o peso corporal e o consumo de alimento e água em ratos (Tese de Doutorado). Universidade de São Paulo, São Paulo, Brasil.

Pessôa, C. V. B., \& Buffara, A. C. L. (2005). Construção de intervalos variáveis de reforçamento em planilha eletrônica de cálculo. Revista Brasileira de Terapia Comportamental e Cognitiva, 3, 133-136. doi: 10.31505/rbtcc.v7i1.48

Rocha, L. M. (2013). Os efeitos da submissão ao Chronic Mild Stress (CMS) no estabelecimento de uma discriminação simples (Dissertação de Mestrado). Pontifícia Universidade Católica de São Paulo, São Paulo, Brasil.

Rodrigues, M. B. (2005). Interações entre o Chronic Mild Stress e o Desempenho Operante: Uma Replicação de Dolabela (2004) (Dissertação de Mestrado). Pontifícia Universidade Católica de São Paulo, São Paulo, Brasil.

Segato, F. N., Castro Souza, C., Segato, E. N., Morato, S., \& Coimbra, N. C. (1997). Sucrose ingestion causes opioid analgesia. Brazilian Journal of Medical and Biological Research, 30, 981-984. doi: 10.1590/S0100879X1997000800011

Thomaz, C. R. C. (2001). O Efeito da Submissão ao Chronic Mild Stress (CMS) sobre o Valor Reforçador do Estímulo (Dissertação de Mestrado). Pontifícia Universidade Católica de São Paulo, São Paulo, Brasil.

Thomaz, C. R. C. (2009). Possíveis inter-relações entre a submissão ao Chonic Mild Stress (CMS) e o desempenho operante (Tese de Doutorado). Instituto de Psicologia da Universidade de São Paulo, São Paulo, Brasil.

Willner, P., Towell, D., Sampson, S., Sophokleous, S., \& Muscat, R. (1987). Reduction of sucrose preference by chronic unpredictable mild stress, and its restoration by a tricyclic antidepressant. Psychopharmacology, 93, 358-364. doi: 10.1007/BF00187257
Willner, P. (1997). Validity, reliability and utility of the chronic mild stress (CMS) model of depression: A 10year review and evaluation. Psychopharmacology, 134, 319-329. doi: 10.1007/s002130050456

Willner, P., Benton, D., Brown, E., Cheeta, S., Davies, G., Morgan, J., \& Morgan, M. (1998). "Depression" increases "craving" for sweet rewards in animal and human models of depression and craving. Psychopharmacology, 136, 272-283. doi: 10.1007/s002130050566

Submetido em: 11/03/2019 Aceito em: 27/06/2019 